\title{
LA QUESTION DE L'ADHÉSION DE CHYPRE À L'UNION EUROPÉENNE ET LE PROBLÈME DE LA RÉPUBLIQUE TURQUE DE CHYPRE-NORD
}

\author{
Semin Suvarierol
}

Le cinquième élargissement de l'Union européenne ${ }^{1}$ engendre de nombreuses difficultés. Mais il est aussi clair que chaque candidature a ses problèmes particuliers. Toutefois, même un regard rapide sur la liste des candidats peut nous indiquer le cas le plus particulier, celui de Chypre. La petite île d'Aphrodite divisée en deux depuis 26 ans constitue donc un de ces milliers de problèmes de I'UE. Le paradoxe est que Chypre est, aux niveaux économique et administratif, le meilleur candidat ${ }^{2}$. Elle est le plus prospère parmi eux et elle dispose de toutes les structures administratives nécessaires. Mais quelle Chypre? C'est la question préoccupante qui se pose à propos de la candidature chypriote. II s'agit d'une candidature politiquement problématique. Le conflit chypriote, demeurant non résolu depuis au moins 37 ans, pèse lourd sur l'adhésion de Chypre à l'UE, tout comme il pèse sur la population de l'île, ainsi que sur tous les autres acteurs qui y sont impliqués.

\section{REPERES HISTORIQUES}

L'Accord d'association entre Chypre et la CEE est signé le 19 décembre 1972 à Bruxelles et entre en vigueur le $1^{\text {er }}$ juin 1973. L'accord prévoyait la réalisation d'une union douanière en deux phases, dont la première était censée prendre fin le 30 juin 1977, tandis que la deuxième devait en principe durer cinq ans. Cet accord devait profiter aux deux communautés de l'île sans pour autant faire de discrimination entre elles. Le coup d'État de

\footnotetext{
${ }^{1}$ Depuis le $1^{\text {er }}$ novembre 1993, la Communauté européenne est devenue l'Union européenne suite à l'entrée en vigueur du traité de Maastricht. On emploiera le terme CEE pour la période antérieure au $1^{\mathrm{er}}$ novembre 1993 et le terme UE pour la période postérieure à cette date.

${ }^{2}$ La stratégie de pré-adhésion pour Chypre lui était propre. II n'y avait pas de feuille de route pour Chypre. Elle bénéficiait d'une assistance technique mais pas du programme Phare, adressé aux PECO (pays de l'Europe centrale et orientale). Cela a récemment changé afin de traiter tous les candidats d'une manière égale. Le programme MEDA fonctionnera désormais comme un instrument de pré-adhésion pour Chypre et Malte. Voir European Parliament, Committee on Foreign Affairs, Human Rights, Common Security and Defence Policy,
} 
la junte grecque suivi par l'intervention turque de 1974 qui ont provoqué une grave crise économique, ont retardé la mise en vigueur de l'union douanière ${ }^{3}$. Après des extensions successives de la première phase, le protocole de la deuxième phase a été signé le 19 octobre 1987 à Luxembourg. Entré en vigueur le $1^{\text {er }}$ janvier 1988, le protocole envisageait la réalisation progressive d'une union douanière sur une période de 15 ans, s'achevant en 2002 ou 2003.

Le 4 juillet 1990, le gouvernement de la République de Chypre dépose sa demande officielle d'adhésion à la Communauté européenne, au nom de l'ensemble de l'île. Lors de sa réunion du 17 septembre 1990 à Bruxelles, le Conseil des ministres décide de transmettre cette demande à la Commission qui rend, sous certaines conditions, un avis favorable pour l'ouverture des négociations d'adhésion avec Chypre le 30 juin 1993. Le Conseil avalise ensuite cet avis lors de son sommet à Luxembourg le 4 octobre 1993. Le Conseil européen de Corfou des 24-25 juin 1994 note que le prochain élargissement de l'Union englobera Chypre et Malte. Cette décision est confirmée par les sommets suivants. C'est grâce au «compromis historique» du 6 mars 1995 que Chypre obtient la garantie que ses négociations d'adhésion commenceront six mois après la fin de la CIG (conférence intergouvernementale) de 1996. En échange de cette promesse, la Grèce lève son veto à l'union douanière avec la Turquie. Comme promis, la décision d'entamer les négociations avec la République de Chypre est prise par le Conseil européen, réuni à Luxembourg les 12-13 décembre 1997. Juste avant le commencement des négociations, le Président chypriote grec, Glafcos Cléridès, invite la communauté chypriote turque à se joindre à l'équipe de négociation. L'invitation formulée le 12 mars 1998, lors de la conférence européenne à Londres, est transmise aux dirigeants chypriotes turcs par la présidence britannique de l'UE. L'invitation refusée, les négociations démarrent formellement le 31 mars 1998 mais ne débutent réellement que le 10 novembre 1998. Lorsque ces négociations avancent, le Sommet d'Helsinki des 10-11 décembre 1999 estime que la résolution du problème de Chypre ne constitue pas un préalable à l'adhésion de Chypre à l'UE, comme l'auraient souhaité certains Etats membres. Ceci constitue le deuxième compromis historique car c'est lors du même sommet que la Turquie se voit reconnaître le statut de « candidat à l'UE » mais sans que les négociations soient ouvertes avec elle (insuffisante observation des droits de l'Homme et des libertés publiques), grâce à la levée du veto grec.

\footnotetext{
Rapporteur: Elmar Brok, Report on the Proposal for a Council regulation on the implementation of operations in the Framework of the pre-accession strategy for Cyprus and Malta, 3 février 2000, p. 19.

${ }^{3}$ La partie nord de l'île rassemblait la plus grande partie de la richesse et du potentiel chypriote en 1974: la moitié de la surface agricole utile, le tiers du potentiel industriel, plus de moitié des ressources minières, l'essentiel des équipements portuaires, les trois quarts de la capacité hôtelière de l'île. Voir Marie-Pierre Richarte, La Partition de Chypre. Etude géopolitique en Méditerranée orientale, Thèse de Doctorat de géographie sous la direction de Paul Claval, Université Paris IV, 1995, p. 219.
} 


\section{UNE CANDIDATURE POLITIQUEMENT PROBLEMATIQUE}

Ce qui rend la candidature chypriote problématique est le fait qu'elle a été formulée par la République de Chypre, le seul État reconnu par l'Union européenne et la communauté internationale ${ }^{4}$ dans une île divisée de facto en deux États souverains. La République turque de Chypre-Nord (RTCN) se trouve donc impliquée dans une demande d'adhésion qu'elle n'a pas formulée. Face à ce problème, I'UE, puisqu'elle ne peut aller à l'encontre du droit international, «dans la logique de sa position constante et cohérente avec celle des Nations unies sur la légitimité du gouvernement de la République de Chypre et la nonreconnaissance de la 'République turque de Chypre Nord', a considéré cette demande comme recevable et déclenché les procédures prévues par les Traités pour examiner cette candidature» ${ }^{5}$.

Une fois le processus d'adhésion démarré, les choses n'apparaissaient plus aussi simples. L'Union s'est trouvée face à un dilemme, car ses valeurs démocratiques ne permettaient pas non plus que le gouvernement chypriote grec qui ne représentait pas les Chypriotes turcs procède aux négociations d'adhésion qui aboutiraient formellement à l'adhésion de l'ensemble de l'île. Des voix se sont élevées pour inclure les Chypriotes turcs en les invitant à joindre la délégation de négociation chypriote.

Malgré l'invitation présentée par le Président de la République de Chypre, on voit encore une fois que le problème de l'adhésion de Chypre n'est pas si facile à résoudre. Une fois l'invitation rejetée par la RTCN, le processus d'adhésion a commencé sans les Chypriotes turcs. Pendant que les négociations avancent avec la République de Chypre, il y a de plus en plus de Chypriotes turcs qui souhaitent participer à ce processus.

La complexité de toute la situation s'inscrit dans le problème non résolu de Chypre. En admettant qu'une solution ne soit pas trouvée, le souhait de voir les Chypriotes turcs participer au processus reste problématique. «Oui, mais comment ?» est la question qui court à Nicosie, à Lefkoşa ${ }^{6}$ et à Bruxelles.

II faut noter premièrement qu'il y a un manque d'information sur les Chypriotes turcs dans la littérature scientifique sur le problème de l'adhésion de Chypre à l'UE; et deuxièmement,

\footnotetext{
${ }^{4}$ La République turque de Chypre-Nord (RTCN), proclamée le 15 novembre 1983, est reconnue seulement par la Turquie tandis que la République de Chypre, qui ne contrôle que la zone Sud et qui ne compte que des Chypriotes grecs, maronites, arméniens et d'autres minorités, est reconnue comme seule légitime par tous les autres États. Le Conseil de sécurité adopte sa Résolution 541 (1983) le 18 novembre 1983, qui déplore la déclaration de ladite sécession, qui considère la proclamation non valide, qui exige son annulation et qui appelle tous les Etats à ne reconnaître aucun autre État que celui de la République de Chypre.

${ }^{5}$ Commission des Communautés européennes, Avis de la Commission sur la demande d'adhésion de la République de Chypre à la Communauté, le 30 juin 1993.

${ }^{6}$ Désormais, on va se référer à Nicosie en parlant de la capitale de la République de Chypre et à Lefkoşa lorsqu'il s'agira de la capitale de la RTCN.
} 
une tendance à associer directement la RTCN avec son président Rauf Denktaş ${ }^{7}$. Partant de ce constat, nous chercherons à montrer comment tout ce processus d'adhésion à l'UE est perçu au Nord de l'île.

\section{LES POSITIONS DES ChypRIOTES TURCS FACE A LA CANDIDATURE DE ChYPRE}

\section{A L'UNION EUROPEENNE}

La première réaction de la RTCN face à la demande d'adhésion à l'UE faite par la République de Chypre était plutôt négative. Celle-ci a été perçue comme une menace existentielle vis-à-vis de l'identité de la communauté chypriote turque ${ }^{8}$. C'était surtout le point de vue du gouvernement de la RTCN et ses arguments qui ont présenté cette candidature comme inacceptable.

La RTCN a vigoureusement contesté la demande d'adhésion présentée par la République de Chypre par un mémorandum adressé au Conseil des ministres des Communautés européennes, le 12 juillet 1990. La Commission européenne résume ainsi cette opposition dans son avis de 1993 : « tout en reconnaissant l'intérêt de la communauté chypriote-turque à faire partie de la Communauté, ces autorités dénient au Gouvernement de la République de Chypre le droit d'engager l'ensemble de Chypre dans une telle démarche. (...) Ces autorités considèrent que, dans les circonstances actuelles la Communauté ne devra pas donner suite à cette demande $"{ }^{9}$. Ce n'était donc pas la perspective de l'adhésion elle-même qui était remise en question, mais la manière dont elle était menée. Les Chypriotes turcs n'avaient pas été consultés et une injustice en découlerait si la CEE acceptait la demande d'adhésion telle quelle. Leur position est fondée sur le Traité de garantie et sur le texte de la Constitution de la République de Chypre de $1960^{10}$. Pour mieux comprendre leur raisonnement, il nous paraît pertinent de présenter aussi les opinions de deux professeurs de droit international, Maurice H. Mendelson ${ }^{11}$ et Christian Heinze ${ }^{12}$, dont les opinions

\footnotetext{
${ }^{7}$ Rauf Denktaş assume les fonctions de président de l'Etat fédéré turc de Chypre puis de la RTCN depuis 1976.

8 Thomas Diez, "Last Exit to Paradise? The EU, the Cyprus Conflict, and the Problematic 'Catalytic Effect'», COPRI (Copenhagen Peace Research Institute) Working Papers, 4/2000, p. 13.

${ }^{9}$ Commission des Communautés Européennes, Avis de la Commission sur la demande d'adhésion de la République de Chypre à la Communauté, op. cit., p. 4.

${ }^{10}$ Le Traité de garantie attribuait à chacun des trois États garants le droit d'agir, conjointement ou séparément, dans le but d'établir l'état des choses créé par le traité. La Constitution sauvegardait l'équilibre entre les deux communautés de l'île.

${ }^{11}$ Maurice H. Mendelson, EU and Cyprus: An Expert View: opinion on the application of Republic of Cyprus to join the European Union, Lefkoşa, 1997.

${ }_{12}$ Dr. Christian Heinze, On the question of the compatibility of the admission of Cyprus into the European Union with international law, the law of the EU and the Cyprus Treaties of 1959/60, Appraisal study presented to the Republic of Turkey, Munich, mars 1997.
} 
juridiques ont été demandées par la Turquie et dont les arguments ont ensuite été avancés auprès de l'UE et de ses Etats membres par la RTCN et la Turquie.

Le mémorandum de la RTCN fonde l'opposition chypriote turque à cette demande sur le fait que la République de Chypre, telle qu'elle était conçue et née en 1960, était fondée sur l'existence des deux communautés distinctes et politiquement égales. Le fait que la communauté chypriote grecque assume le titre de République de Chypre ne donne pas le droit à cette communauté de représenter l'ensemble de Chypre, surtout pas dans une démarche aussi fondamentale que l'adhésion à la CEE. Les Chypriotes turcs s'opposent à cet acte unilatéral commis par les Chypriotes grecs avec le titre illégitime de République de Chypre car il constitue une action claire et inacceptable de coercition de leur volonté politique sur les Chypriotes turcs à propos d'une question qui va affecter les vies des générations à venir. Ils doivent avoir une voix sur la question de l'adhésion du pays à la CEE selon l'article 57 de la Constitution reconnaissant au Président (chypriote grec) et au VicePrésident (chypriote turc) un droit de veto sur toute décision de politique étrangère, veto que le Vice-Président ne peut plus exercer depuis décembre 1963.

Aux termes de l'article 185 de la Constitution, texte fondateur, non susceptible d'amendement, l'union totale ou partielle de l'lle avec tout autre État est exclue. Mendelson rappelle que l'intention des traités établissant la République de Chypre était d'éviter la possibilité de procurer à la Grèce ou à la Turquie une position économique plus favorable que l'autre qui reviendrait à une sorte d'“enosis ${ }^{13}$ économique ». C'est pourquoi l'article 8 de la Constitution stipulait que «Le Président et le Vice-Président auront séparément et conjointement le droit de veto définitif sur toute loi ou décision se référant aux affaires de la participation de la République de Chypre à des organisations internationales et pactes d'alliance dont la Grèce et la Turquie font toutes deux parties....». Cela veut dire que le Président et le Vice-Président gardent un droit de veto sur l'adhésion aux organisations dont seul un de ces deux États est membre, ce qui inclut bien entendu l'UE. L'égalité entre les deux mères patries constitue une base aussi importante que l'égalité politique des deux communautés chypriotes ${ }^{14}$. Mendelson conclut alors que l'Administration chypriote grecque n'a pas le droit de demander l'adhésion à l'UE, ni d'en devenir un membre si la Turquie n'en est pas membre.

Le mémorandum soutient que l'union des Chypriotes grecs avec la Grèce, même à travers la CEE, n'est permise ni par la Constitution, ni par le traité de garantie. L'admission aux Communautés européennes des Chypriotes grecs sous le titre de République de Chypre donnerait en effet un deuxième vote à la Grèce dans toutes les délibérations de la CEE car liée spirituellement à la Grèce, la communauté chypriote grecque s'alignerait

\footnotetext{
${ }^{13}$ L'unification de l'île avec la Grèce.

${ }^{14}$ Rauf R. Denktaş, Kıbrıs Meselesinde Son Durum, Kıbrıs, Raif Denktaş Eğitim Vakfı Yayınları, 1996, p. 5.
} 
systématiquement sur les positions de la Grèce au sein des organes européens. C'est seulement en faisant adhérer Chypre, avec un système de gouvernement établi sur l'existence des deux communautés de l'île, que cette éventualité pourrait être évitée. Par ailleurs, la République de Chypre accorde le traitement de la nation la plus favorisée aux trois pays garants. Alors, dit Mendelson, la Grèce et le Royaume-Uni, comme pays garants de Chypre, sont dans l'obligation d'éviter cette adhésion, car si Chypre devient membre de I'UE, il en résultera que la Grèce et le Royaume-Uni recevront un traitement plus favorable que la Turquie qui n'est pas membre de l'UE.

L'adhésion à la Communauté, d'autant qu'elle demande un degré de participation de ses membres, est impraticable dans les circonstances actuelles, car en attendant une solution compréhensive et mutuellement acceptable pour établir une fédération bi-communale et bizonale, Chypre demeure une île divisée par une frontière qui sépare les deux secteurs de l'île. L'unité de Chypre suggérée par la demande de la République de Chypre n'est soutenable ni dans le droit, ni dans les faits. L'analogie que font les Chypriotes grecs entre le cas de Chypre et de l'Allemagne est rejetée par les Chypriotes turcs ${ }^{15}$. Ils avancent que la République fédérale d'Allemagne ne prétendait pas que les limites territoriales de son appartenance à la CEE s'étendaient au-delà de la partie sous son contrôle : elle ne disait pas représenter l'Allemagne de l'Est lorsqu'elle devint membre. II est évident que les Chypriotes grecs ne cherchent pas à limiter leur demande d'une manière comparable.

Mendelson maintient qu'il existe aussi des obstacles légaux à cette adhésion sur le fond du droit communautaire européen. Heinze discute ce point en disant que le gouvernement de Nicosie ne serait pas dans une position de remplir ses obligations envers les autres pays membres. En même temps, les membres seraient confrontés à des difficultés en remplissant leurs obligations à l'égard de l'ensemble de l'île. Heinze ajoute que l'UE serait non seulement justifiée, mais même obligée d'appliquer sa législation sur la totalité du territoire chypriote, si nécessaire en utilisant son pouvoir exécutif. Selon certains I'Union s'exposerait aussi, dans le cadre de la PESC (Politique étrangère et de sécurité commune), à la possibilité de participer aux actions militaires contre la RTCN.

Le mémorandum chypriote turc se réfère aussi à la résolution du Conseil de Sécurité du 12 mars 1990 qui «demande aux parties intéressées de s'abstenir de tout acte qui pourrait aggraver la situation ${ }^{16}{ }^{16}$, soutenant que cet acte détruit l'égalité de statut entre les deux communautés, principe qui constitue le fondement essentiel de la suite des négociations sur la question chypriote. Ainsi, l'adhésion à la CEE d'un État uniquement chypriote grec ne peut

\footnotetext{
${ }^{15}$ L'exemple allemand ne peut être comparé au cas chypriote aux yeux des Chypriotes turcs. En Allemagne, il s'agissait d'un seul peuple, alors qu'à Chypre il y a deux communautés.

${ }^{16}$ Résolution 649(1990) du Conseil de Sécurité du 12 mars 1990, Article 5.
} 
contribuer à la solution des différends entre les deux communautés. Au contraire, cela perpétuera la division de l'île.

L'opposition à cette demande d'adhésion est donc d'une nature fondamentale. Le caractère illégal de l'autorité faisant la demande, la portée de cette demande et les conditions dans lesquelles elle a été présentée rendent la demande nulle et non avenue. La demande n'existe donc pas et ne crée aucune obligation légale ou politique sur la communauté chypriote turque. Par conséquent, la bonne ligne de conduite pour le Conseil serait de ne prendre aucune action sur la demande. "Dans la mesure où la question de la souveraineté du candidat lui-même n'est pas réglée, qui va transférer quelle souveraineté à qui?», se demande-t-on ${ }^{17}$.

Néanmoins, le mémorandum explique aussi que la RTCN ne désire pas créer l'impression qu'elle est opposée à l'adhésion éventuelle d'un État de Chypre, restauré légalement par règlement négocié entre les deux parties égales de l'île. Dès que ce règlement verra le jour, la communauté chypriote turque ne sera pas moins désireuse de voir Chypre devenir membre de la CEE, d'une manière qui va assurer que les bénéfices et les responsabilités de cette adhésion seront partagées par les deux communautés sans discrimination. Les Chypriotes turcs demandent ainsi que justice soit rendue.

Décision prise à Bruxelles, le processus d'adhésion est en cours depuis plus de deux années, mais ce discours officiel du gouvernement de la RTCN existe toujours. La question de l'adhésion à l'UE n'est pas actuellement sur l'agenda de la RTCN, car il n'existe pas de demande d'adhésion faite par elle. Ce qui existe est une demande d'adhésion faite par «l'Administration chypriote grecque» ${ }^{18}$, retenue par l'UE d'une façon injuste et inacceptable. Telle qu'elle existe aujourd'hui, la perspective d'adhésion signifie une attraction économique, mais aussi une menace pour la sécurité et les droits politiques de la communauté chypriote turque ${ }^{19}$.

Pourtant, bien que la position officielle soit fondée sur des arguments juridiques solides, elle semble plutôt caduque aujourd'hui aux yeux de la population chypriote turque. Le quotidien le plus diffusé de la RTCN, Kıbrıs, remarquait, en effet, le 30 mars 2000 que, selon les sondages, les objectifs prioritaires de la grande majorité des Chypriotes turcs sont la résolution du conflit chypriote et l'adhésion à l'UE. En effet, les visages de la scène politique se montrent conscients de cette tendance. Ces deux thèmes majeurs dominaient la dernière élection présidentielle du 15 avril 2000. Même les deux candidats présidentiels connus pour leur hostilité envers I'UE, le Président Rauf Denktaş et le Premier ministre, Derviş Eroğlu,

\footnotetext{
${ }^{17}$ Halûk Kabaalioğlu, «Cyprus and the European Union: A Perspective» in Emel Doğramacı, William Haney et Güray König, Proceedings of the First International Congress on Cypriot Studies, Gazimağusa (Famagouste), Eastern Mediterranean University Press, 1997, p. 220.

${ }_{18}$ La RTCN et la Turquie soutiennent que le gouvernement chypriote grec n'a pas droit à l'appellation « République de Chypre ».
} 
chef de l'UBP (Ulusal Birlik Partisi, «Parti de l'unité nationale»), ont cherché pendant toute la campagne à convaincre l'électorat qu'ils n'étaient pas opposés à l'adhésion à l'UE ${ }^{20}$.

On peut constater avec confiance qu'aujourd'hui la question européenne intéresse véritablement les Chypriotes turcs. Mais ce n'était pas le cas au début, en 1990 par exemple, lorsque la République de Chypre avait déposé sa candidature ${ }^{21}$. L'exception était la vision d'Alpay Durduran, figure marginale et marginalisée de la politique chypriote turque ${ }^{22}$. Dirigeant actuellement du YBH (Yurtsever Birlik Hareketi, «Mouvement de l'union patriotique»), il avait déjà mis la question de l'UE sur son agenda lors de l'élection présidentielle du 22 avril 1990. II disait à l'époque ${ }^{23}$ «Venez, résolvons le problème chypriote et devenons membre de I'UE! Devenons européens! Prenons un passeport rouge et voyageons en Europe! $!^{24}$ » Durduran avoue que les gens ne se souviennent pas de toutes les explications qu'il avait données à l'époque mais uniquement du passeport rouge qu'il tenait dans ses mains lors de sa propagande. Malheureusement, ce n'est qu'aujourd'hui qu'on lui donne raison pour avoir prévu l'importance de l'adhésion à l'UE. «Suis-je un clairvoyant?» se moque-t-il, reprochant au public d'ignorer encore ce que signifie l'UE. Mais au moins, veulent-ils maintenant majoritairement adhérer à l'UE.

La montée de l'enthousiasme pour l'UE est encore plus forte depuis le sommet d'Helsinki où la candidature de la Turquie a été reconnue. On n'exagère guère si on dit que la vie de «l'enfant patrie» (yavruvatan) est liée à ce qui se passe dans la mère patrie (anavatan) ${ }^{25}$. Après tout, la Turquie est «la fenêtre unique des Chypriotes turcs sur le monde» ${ }^{26}$ du fait de la non-reconnaissance de la RTCN qui l'isole politiquement et économiquement. Alors depuis Helsinki, même les plus réticents à l'égard de l'Europe envisagent la perspective d'adhésion avec plus d'optimisme, même si c'est toujours un optimisme réservé.

Quant à la représentation de l'adhésion à l'UE dans les esprits chypriotes turcs, elle est très variable. D'un côté, dans son discours assez particulier, Denktaş la décrit depuis 1990 avec toutes sortes de noms : " un piège ou une intrigue byzantine » (Bizans tuzağı/oyunu), « un train grec » (Yunan treni), « une machine à hâcher » (kıyma makinesi). C'est un piège,

\footnotetext{
${ }^{19}$ Entretien avec Ergün Olgun, Conseiller politique du Président de la RTCN, Lefkoşa, 5 avril 2000.

${ }^{20}$ Nos entretiens et discussions avec les Chypriotes turcs nous ont donné la conviction que beaucoup d'entre eux ne croyaient guère aux discours de ces deux personnalités sur l'UE, disant qu'ils ne faisaient que de la «propagande artificielle» pour sauver la face contre tous les autres candidats qui étaient pour l'adhésion.

Entretien avec İsmail Bozkurt, Président du Centre des Études chypriotes de la DAÜ (Doğu Akdeniz Üniversitesi, «l'Université de la Méditerranée orientale»), Gazimağusa (Famagouste), 5 avril 2000.

22 II est marginal au sens où ni lui, ni son parti politique, n'ont obtenu plus de $3 \%$ des votes lors des différentes élections. Il a été marginalisé par le fait qu'il reste le seul dirigeant qui s'oppose avec constance à Rauf Denktaş et à la Turquie. Pour les résultats des élections en RTCN, voir Annexe.

${ }^{23}$ Toutes les informations qui suivent résultent de l'entretien avec Alpay Durduran, Lefkoşa, 6 avril 2000.

${ }^{24}$ Les Chypriotes turcs ne peuvent voyager, à l'exception de la Turquie bien entendu, qu'avec le passeport turc. En Turquie, les passeports diplomatiques sont de couleur rouge, d'où la référence au «passeport rouge».

${ }^{25}$ Ce discours unique de yavruvatan-anavatan constitue le cœur de la socialisation politique des enfants à l'école à la RTCN et en Turquie. On apprend alors que les relations entre les deux ne sont qu'étroites et chaleureuses.

${ }^{26}$ Entretien avec Tahsin Ertuğruloğlu, Ministre des affaires étrangères et de la défense de la RTCN, Lefkoşa, 7 avril 2000.
} 
une intrigue byzantine, car sans une résolution complète de la question chypriote, l'adhésion à l'UE signifie «la multiplication par zéro de tous les droits et les pouvoirs obtenus en 1960, l'intégration de Chypre avec la Grèce à travers l'UE et l'annulation de tous les droits et les pouvoirs que la Turquie a sur Chypre provenant des traités de $1960{ }^{27}$. Ainsi ce que les Chypriotes grecs ou autres décrivent comme le train vers l'UE qui s'enfuit n'est qu'un train grec. Les Chypriotes turcs peuvent seulement y monter quand il sera un train chypriote. Sinon, ils entreraient dans une machine à hâcher ${ }^{28}$. Ce discours qu'on est susceptible d'entendre des gens de la génération de Denktaş a ses origines dans la mémoire des années 1963-1974 ${ }^{29}$. Toutes ces années de difficultés, d'atrocités, l'exemple des turcs en Crète ou en Thrace occidentale ou du sort des minorités dans les Balkans font qu'ils ne font guère confiance aux Chypriotes grecs, aux Grecs ou aux forces internationales de protection. Le but hellène est toujours le même : faire de Chypre une île hellène et parvenir à l'enosis. Ils ont vécu à quel point l'ONU n'a pu les protéger depuis 1964 . Ils ont vu après la fin de la guerre froide, combien de temps les puissances occidentales mettent pour intervenir en cas de génocide et combien de gens meurent entre-temps ${ }^{30}$.

De l'autre côté, pour la grande majorité des Chypriotes turcs, l'UE représente une grande opportunité à ne pas manquer, voire une «dernière porte d'espoir (son umut kapısı)» ${ }^{31}$. Tout d'abord, ils en ont vraiment assez des complications créées ${ }^{32}$ par le conflit chypriote. Ils ont hâte de sortir de cet isolement du reste du monde. Ils ne veulent surtout pas que leurs enfants subissent ces conditions. D'autre part, ils savent ce que signifient des niveaux de vie élevés. Ayant vécu sous l'administration britannique, le but du Chypriote turc dans la vie est d'avoir toujours un niveau de vie plus haut ${ }^{33}$. L'adhésion à l'UE évoque ainsi le rêve des standards/valeurs européens, qui inclut la stabilité et la paix qu'elle va apporter. Après tout, tout comme pour leurs compatriotes chypriotes grecs, Chypre fait partie de l'Europe et doit

\footnotetext{
${ }^{27}$ Denktaş, op. cit., p. 8.

28 Yeni Demokrat, mercredi 5 avril 2000.

${ }^{29}$ Pour un récit de ces jours avec des photographies, voir Çiler İncirli, Bir daha asla, Lefkoşa, Kuzey Kıbrıs Türk Cumhuriyeti Dışişleri ve Savunma Bakanlığı (Le ministère des Affaires étrangères et de la défense de la République turque de Chypre-Nord), 1998.

${ }^{30}$ «Nous sommes 120000 ici. En Bosnie, 120000 sont morts en deux mois sans que l'Europe bouge. Lui feraisje confiance en entrant dans l'UE? Prendrais-je le risque de renoncer à la garantie efficace de la Turquie?» Entretien avec Doç. Dr. Hasan Ali Bıçak, Professeur, Responsable du Centre d'information et de recherche sur I'Union européenne à la DAÜ, Gazimağusa, 7 avril 2000.

${ }^{31}$ Entretien avec Sevgül Uludağ, Journaliste au Yenidüzen, Participante aux activités bicommunautaires, Lefkoşa, 7 avril 2000.

32 Les gens sont confrontés à des problèmes liés à la non-reconnaissance de leur État dans leur vie quotidienne. Un commerçant doit expliquer à ses homologues européens que leur adresse se finit par Mersin 10, Turkey (un code inventé après 1974- le courrier de la RTCN est ainsi transféré par la poste turque du port de Mersin à la RTCN), mais qu'ils sont en fait au Nord de Chypre; qu'ils ne peuvent pas faire de transaction à travers des bureaux à la République de Chypre car il y a un blocus commercial; qu'ils ne peuvent non plus faire la transaction à travers des bureaux en Turquie car ils sont à Chypre. Entretien avec Emine Erk, Avocat, Participante aux activités bicommunautaires, Lefkoşa, 8 avril 2000.

${ }^{33}$ Entretien avec Fatma Azgın, Pharmacienne, Participante aux activités bicommunautaires, Lefkoşa, 6 avril 2000.
} 
être membre de l'Union ${ }^{34}$. Et «le train de l'UE est bien en route», les Chypriotes turcs n'ayant plus la force d' attendre d'être à bord ${ }^{35} \ldots$

La question pertinente et préoccupante que les Chypriotes turcs se posent à présent est celle des conditions auxquelles ils pourraient ou aimeraient adhérer à l'UE. Les scénarios d'adhésion qu'ils envisagent prennent en compte deux critères : la résolution du problème chypriote; l'adhésion de la Turquie à l'UE.

Manifestement, tout le monde préfère d'abord voir la solution de la question chypriote, puis l'adhésion à I'UE. Ceci est très important, parce que c'est la solution du conflit qui déterminera le statut des Chypriotes turcs, et qui restituera leur titre de partenaire égal aux Chypriotes grecs. Ils se rendent compte du fait que les États sont les seuls vrais interlocuteurs de l'Union ${ }^{36}$. Les termes de cette solution assureront aussi leur sécurité et permettront d'imposer des dérogations concernant les trois libertés fondamentales : liberté de circulation, d'établissement, droit de propriété ${ }^{37}$. Faute de solution, personne ne peut garantir la paix et la stabilité sur l'île.

II faut préciser ici ce que les Chypriotes turcs entendent par aboutir à une juste solution du problème chypriote. La position officielle du gouvernement de la RTCN, adopté par la droite chypriote turque ${ }^{38}$, est en faveur d'une solution confédérale. Pour eux la reconnaissance est indispensable. Avec un ton nationaliste, ils disent que le drapeau de la RTCN ne peut être baissé. Pour les partis de gauche, «Peu importe le nom!» de la nouvelle formation. Ils sont d'avis que les éléments d'une solution sont évidents: deux entités politiques égales et autonomes se gouvernant séparément à l'intérieur, mais ayant une seule représentation internationale. Le YBH reste le seul «partisan d'une solution fédérale telle qu'elle a été acceptée par la classe politique chypriote grecque et l'ONU» ${ }^{39}$, c'est-à-dire avec un gouvernement fédéral fort, et «rejette l'hypothèse confédérale aussi bien que la demande de reconnaissance internationale de la $[\mathrm{RTCN}]{ }^{40}$. Tous pensent que la solution maintiendra la garantie de la Turquie sur la sécurité de Chypre ${ }^{41}$.

Lorsqu'il s'agit de la compatibilité de la solution de la question chypriote avec les normes européennes, ils citent des exemples des dérogations relatives surtout aux trois libertés

\footnotetext{
${ }^{34}$ Entretien avec Ferdi Sabit Soyer, Secrétaire général du CTP (Cumhuriyetçi Türk Partisi, «Parti républicain turc»), Lefkoşa, 6 avril 2000.

${ }_{35}$ Avrupa, mercredi 5 avril 2000.

${ }^{36}$ Entretien avec Ali Erel, Membre de la K.K.T.C. IŞAD (KKTC Işadamları Derneği, «l'Association des Hommes d'affaires de la RTCN»), Lefkoşa, 6 avril 2000.

${ }^{37}$ Les Chypriotes turcs craignent toujours la domination chypriote grecque, surtout au niveau économique. C'est pourquoi ils demandent au moins des périodes transitoires après la résolution du conflit.

${ }^{38}$ Les partis politiques de la droite tiennent 37 sièges sur un total de 50 sièges au Parlement de la RTCN.

${ }^{39}$ Gilles Bertrand, «Vingt-cinq ans après, où en est la partition de Chypre?», Les Études du CERI, (59), novembre 1999, p.22.

${ }^{40}$ Idem.

${ }^{41}$ Les Chypriotes turcs ont un attachement émotionnel et psychologique à l'armée turque. Selon Christos $P$. loannides, elle est vue comme un «permanent guardian angel» et la RTCN est l'enfant des militaires turcs. Voir
} 
fondamentales dans des pays membres ${ }^{42}$. Ils ont confiance dans le fait que l'UE est prête à approuver toute solution. Le défi reste, comme toujours, de pouvoir arriver à une solution avec les Chypriotes grecs.

La présidence, I'UBP et le DP (Demokrat Parti), dirigé par Salih Coşar, à droite et le TKP (Toplumcu Kurtuluş Partisi, «Parti populiste/ communautaire de libération »), dont le leader, Mustafa Akıncı, est le vice-premier ministre depuis décembre 1998, à gauche, soutiennent l'adhésion après la résolution de la question chypriote. Le CTP (Cumhuriyetçi Türk Partisi, «Parti républicain turc»), le parti le plus ancien de la gauche dirigé par Mehmet Ali Talat, est d'avis que les deux processus des négociations (celui en vue de la résolution du conflit et celui en vue de l'adhésion à l'UE) doivent être menés simultanément afin que la RTCN puisse adhérer à l'Union dès la solution atteinte. Selon le $\mathrm{YBH}$, il ne faut pas perdre de temps. L'adhésion peut donc avoir lieu avant même qu'une solution soit adoptée.

Faut-il alors attendre que la Turquie ait adhéré à l'UE ? La gauche est unanimement d'avis qu'une fois la solution déterminée, il n'y a guère besoin d'attendre la Turquie. Comme cette solution va sûrement sauvegarder les droits de la Turquie en tant qu'Etat garant, il n'y a rien à craindre. La Turquie est un pays immense avec des problèmes immenses à résoudre avant d'adhérer à l'UE alors que l'adaptation du nord de Chypre aux critères d'adhésion peut se faire dans deux à trois ans. Pourquoi attendre l'adhésion de la Turquie qui va prendre au moins dix à quinze ans? En plus, ils ne pensent pas que I'UE ferait attendre les Chypriotes grecs autant ${ }^{43}$ car ils seront bientôt prêts à l'adhésion. La présence des Chypriotes turcs serait bénéfique aussi à la Turquie, car ils représenteraient une voix en faveur de la Turquie.

Les autres sont partagés sur cette question. Le DP apparaît comme étant le parti de droite le plus flexible. Maintenant que la Turquie est plus proche de l'adhésion à l'UE depuis le Sommet d'Helsinki, il n'est plus réaliste d'insister pour que Chypre réunifiée doive attendre l'adhésion de la Turquie ${ }^{44}$. À condition que la Turquie possède les mêmes droits que la Grèce à Chypre, sur des questions militaires et économiques, Chypre peut entrer dans une

\footnotetext{
Christos P. Ioannides, In Turkey's Image: The Transformation of Occupied Cyprus into a Turkish Province, New Rochelle, NY, Caratzas, 1991, pp. 164-165.

${ }^{42}$ Ils se réfèrent particulièrement à l'exemple des îles finlandaises autonomes d'Âland, dont le peuple et la langue officielle sont suédois. Par exemple, il est nécessaire de disposer de la citoyenneté locale ålandaise pour : voter et être éligible aux élections législatives du Lagting ; acquérir et posséder des biens immobiliers en Åland; faire du commerce en Åland. Les limitations dans l'acquisition d'immobiliers sont créées afin de garder les terres aux mains des Ålandais. Les rapports spéciaux entre l'Union européenne et Åland sont rédigés dans un protocole d'accord annexé au Traité d'adhésion à l'Union. Selon le protocole, bien qu'Åland soit un membre de I'Union européenne, elle est en dehors des accords concernant les taxes. Åland a la garantie de conserver ses conditions particulères relatives à l'achat de biens fonciers et des activités commerciales dans la province. Enfin une mention dans le protocole confirme le statut spécial d'Åland dans le droit international. Voir $<$ http://www.aland.fi/virtual/fransk/frame.html>.

${ }^{43}$ Discours de Mustafa Akıncı lors de la Conférence intitulée «Kıbrıs'ta Çözüm ve Avrupa Birliği», AKM, Lefkoşa, 5 avril 2000.

${ }^{44}$ Entretien avec Hakkı Atun, Président honoraire du DP, Ancien Premier Ministre et Président du Parlement de la RTCN, Lefkoşa, 7 avril 2000.
} 
Union sans la Turquie. Ce recours aux termes réalistes est dominant dans le discours de la droite chypriote turque. Selon cette vision, qui définit aussi la politique étrangère de la RTCN, les rapports de force entre la Turquie et la Grèce doivent être toujours conservés. De plus, adhérer à une Union dont la Grèce est membre mais pas la Turquie est menaçant au sens où les intérêts chypriotes turcs ne seraient pas protégés ${ }^{45}$. II est clair que Chypre aura très peu de représentants au sein de l'Union élargie. Alors que les intérêts chypriotes grecs pourraient être défendus par la Grèce, les Chypriotes turcs n'auraient pas la même opportunité $^{46}$. La possibilité d'une intervention turque à Chypre en tant que territoire de I'Union deviendrait problématique ${ }^{47}$. Ainsi, I'UBP maintient, par exemple, que cette décision serait à prendre en consultation avec la Turquie ${ }^{48}$. Ergün Olgun, le conseiller du président, affirme qu'il faut attendre jusqu'à ce qu'une perspective définitive pour l'adhésion (comme par exemple, une garantie que la Turquie deviendrait membre dans une période de six mois) soit donnée à la Turquie. De toute façon, personne ne peut dire que la RTCN est plus prête que la Turquie à l'adhésion aujourd'hui. La Turquie a au moins commencé les efforts d'harmonisation; les Chypriotes grecs y travaillent depuis des années, mais la RTCN en est encore loin ${ }^{49}$.

Il existe un dernier scénario d'adhésion auquel personne ne croit ou ne veut croire: l'adhésion seule des Chypriotes grecs. Tout d'abord, ce n'est pas du tout l'intérêt de l'UE d'importer un tel conflit. Ils croient que ce scénario obligerait les Chypriotes grecs à définir les limites de leur territoire pour faire savoir à l'UE où l'acquis communautaire serait appliqué. Cela, disent Olgun et Ertuğruloğlu, les Chypriotes grecs ne l'accepteraient jamais, car leur but est de garder "l'intégralité de cette île hellène». Ils ajoutent que la Turquie est beaucoup trop importante pour l'UE, et que I'Union ne risquerait donc pas d'éloigner la Turquie pour les Chypriotes grecs. Mais si cela arrive, la division de Chypre deviendra perpétuelle. De son côté Alpay Durduran pense que les gouvernements nationalistes comme ceux de la République de Chypre «donnent le mal de tête à l'UE». Il est d'avis que la Grèce pose déjà des problèmes aux Européens et qu'ils ne voudraient pas d'un tel deuxième État membre.

Les autres en sont moins sûrs. Pour eux, c'est le pire scénario possible. C'est le «cauchemar». Ce scénario produirait une catastrophe pour la communauté chypriote

\footnotetext{
${ }^{45}$ Kabaalioğlu, op. cit., p. 223.

${ }^{46}$ II est même défendable qu'aujourd'hui les représentants grecs soutiennent la cause chypriote grecque au sein de l'Union, alors que la cause chypriote turque reste non entendue. Voir Michael Stephen, The Cyprus Question, London, British-Northern Cyprus Parliamentary Group, 1997, p. 67. Pour les Chypriotes turcs, la candidature de la Turquie représente aujourd'hui plus d'opportunités à se faire entendre par les Européens. La réception de Denktaş par le ministre des Affaires étrangères allemand, Joschka Fischer suite au sommet d'Helsinki, par exemple, a été interprétée en RTCN comme un signe positif de la volonté européenne d'écouter les Chypriotes turcs.

${ }^{47}$ Kabaalioğlu, op. cit., p. 229

${ }^{48}$ Entretien cité avec Tahsin Ertuğruloğlu.

49 Idem.; entretien avec Ergün Olgun.
} 
turque, car leur statut serait rétrogradé par rapport à celui de 1960. Les Chypriotes turcs deviendraient individuellement les sujets de I'UE, ce qui ferait que tout le monde pourrait obtenir un passeport chypriote pour émigrer en Europe. Mustafa Akıncı dit que cela produirait «15 Londres au lieu d'un Londres» ${ }^{50}$. La population chypriote turque de l'île risquerait donc de diminuer jusqu'à l'extinction. Donc, il faut faire tout ce qui est nécessaire pour éviter ce scénario, étant donné que le «chantage grec» de bloquer l'élargissement de I'UE le rend tout à fait plausible ${ }^{51}$.

Que peuvent faire les Chypriotes turcs à l'heure actuelle alors que le processus d'adhésion progresse à grands pas avec les Chypriotes grecs? Le gouvernement de la RTCN a refusé l'invitation de participer aux négociations d'adhésion au sein de la délégation chypriote car cette participation correspondrait à une reconnaissance de la République de Chypre. L'acceptation signifierait la multiplication par zéro de toute leur cause/lutte nationale.

II est donc toujours hors de question de se joindre à cette délégation qu'ils appellent la délégation chypriote grecque. «Qui serions-nous au sein de cette délégation?», beaucoup d'entre eux se le demandent. La réponse est simple: une simple minorité. Ils sont convaincus que les Chypriotes grecs, étant les seuls reconnus par l'UE, prendraient les décisions, et eux, comme minoritaires, seraient soumis aux décisions de la majorité chypriote grecque. C'est cette vision qui avait incité le Président Denktaş à demander la reconnaissance de la RTCN comme pré-condition de la participation des Chypriotes turcs à la délégation chypriote.

Insistant sur l'égalité politique, le gouvernement de la RTCN a demandé une voie séparée et parallèle de négociations avec l'UE ${ }^{52}$ lors de la dernière visite de Günter Verheugen à Chypre, bien que ce dernier ait bien précisé qu'il n'y aurait pas une deuxième négociation pour les Chypriotes turcs. Le CTP, considérant que l'Union ne reconnaîtra jamais la RTCN, veut absolument qu'on trouve un moyen pour y participer. Mehmet Ali Talat promettait lors de la dernière campagne électorale qu'il recevrait des mains de Georges Vassiliou ${ }^{53}$ le dossier des chypriotes turcs si ce dernier venait à être élu ${ }^{54}$. Pour lui et son parti, l'UE reconnaît déjà les Chypriotes turcs comme des partenaires égaux des Chypriotes grecs. Après tout, cette participation pourrait avoir lieu en adoptant la formule des négociations sous l'égide de l'ONU où les dirigeants sont présents comme les leaders des deux

\footnotetext{
${ }^{50}$ La plupart des émigrés chypriotes turcs résident à Londres.

${ }^{51}$ Entretien cité avec Ferdi Sabit Soyer.

52 La conduite de négociations parallèles avec, d'un côté, les Chypriotes grecs et, d'un autre côté, la communauté chypriote turque impliquerait la reconnaissance de la RTCN. Ceci s'opposerait à la ligne suivi jusque là par l'UE. Voir Corine Tonneau, Le rapprochement Chypre-Union européenne: l'impact du conflit intercommunautaire, Mémoire de fin d'études, Université Catholique de Louvain, 1999, p. 74.

${ }^{53}$ Georges Vassiliou est le chef de la délégation de la République de Chypre dans les négociations avec l'UE.

54 Yenidüzen, mercredi 5 avril 2000.
} 
communautés $^{55}$. Quant au YBH, Alpay Durduran, s'il était au pouvoir, rejoindrait immédiatement les négociations, demandant d'abord qu'on en discute les modalités. De toute manière, dit-il, ces négociations ne portent que sur les aspects techniques de l'adhésion à l'UE. Comment I'UE peut-elle aborder des questions qui concernent uniquement les Chypriotes turcs avec les Chypriotes grecs?

Surtout depuis Helsinki, les Chypriotes turcs se sont rendus compte qu'ils sont plus que jamais dans le vide. Chypriotes grecs et Turquie sont tous deux en train de se préparer pour l'adhésion à l'UE. «ll est temps que nous commencions les préparatifs aussi», disent-ils unanimement. Ils ont vraiment besoin de s'informer sur ce qu'implique une adhésion à l'UE. L'opinion publique et les partis d'opposition trouvent que le gouvernement ne fait aucun effort là-dessus. Pourtant, il faudrait vraiment mettre en route l'harmonisation, sinon ils perdront encore du temps pour rattraper le Sud ou la Turquie.

Déjà, ceux qui soutiennent l'adhésion à l'UE, c'est-à-dire les partis de la gauche et les groupes de la société civile chypriote turque, notamment les syndicats et les hommes d'affaires, ont des contacts avec des responsables de I'UE ou avec leurs homologues en Europe. Les participants aux activités bicommunautaires sont parmi les mieux informés sur I'UE, grâce à ces réunions. Les syndicats échangent des informations liées à leurs domaines avec leurs homologues et informent leurs membres sur l'UE à travers des bulletins d'informations. Les responsables de la confédération des syndicats des employés, TÜRK$\mathrm{SEN}^{56}$, qui réunit en son sein 13 des 36 syndicats d'employés ${ }^{57}$, KTÖS (Kıbrıs Türk Öğretmenler Sendikası, "Syndicat des enseignants chypriotes turcs») ${ }^{58}$, KTOEÖS (Kıbrıs Türk Orta Eğitim Öğretmenler Sendikası, «Syndicat chypriote turc des enseignants du sécondaire») ${ }^{59}$, indiquent tous qu'ils soutiennent l'adhésion à l'UE, le plus tôt possible, répétant toujours : on ne peut plus supporter ces conditions. La KKTC IŞAD (KKTC Işadamları Derneği, «l'Association des Hommes d'affaires de la RTCN») ${ }^{60}$ organise souvent des ateliers consacrés au sujet de l'adhésion. Ils y discutent les avantages et les désavantages que I'Union peut apporter aux Chypriotes turcs. Ils invitent les responsables du bureau de la Commission européenne à Nicosie.

La société civile chypriote turque est très développée surtout si on tient compte de la taille de la RTCN. Surtout les syndicats dont les membres incluent les fonctionnaires de l'État y ont une influence certaine. Il est donc tout à fait positif qu'il y ait au moins un effort visible de

\footnotetext{
${ }^{55}$ Entretien cité avec Emine Erk.

${ }^{56}$ Entretien avec Önder Konuloğlu, Président de TÜRK-SEN, Lefkoşa, 6 avril 2000.

${ }^{57}$ Clement H. Dodd, «Political and Administrative Structures», in Clement H. Dodd (ed.), The Political Social and Economic Development of Northern Cyprus, Huntingdon, The Eothen Press, 1993, p. 179.

${ }^{58}$ Entretien avec Varol Öztuğ, Secrétaire général du KTÖS, Lefkoşa, 8 avril 2000.

${ }^{59}$ Entretien avec Ahmet Barçın, Président du KTOEÖS, Lefkoşa, 8 avril 2000.

${ }^{60}$ Entretien avec Ali Erel et Mustafa Damdelen, Membres de la KKTC işAD, Lefkoşa, 6 avril 2000.
} 
leur part visant la perspective d'adhésion à l'UE. Mais finalement, c'est le gouvernement qui doit prendre les mesures substantielles et informer les citoyens globalement. Le premier ministre a aussi annoncé pendant sa campagne électorale qu'il avait établi un comité pour la préparation à l'adhésion. On n'en a pas entendu parler depuis. Si le but est vraiment d'informer, remarque le ministre des Affaires étrangères, l'UE peut renseigner les Chypriotes turcs à travers le bureau de la Commission européenne à Ankara plutôt qu'à travers celui à Nicosie.

Passé l'épisode scandaleux des élections présidentielles ${ }^{61}$, les Chypriotes turcs ont un seul souci majeur : la situation de leur économie. Leur système fragile ${ }^{62}$ qui ne tenait que grâce à l'aide financière turque, a volé en éclats suite à la crise bancaire ${ }^{63}$. L'embargo international exercé sur la RTCN touche de plein fouet les Chypriotes turcs. Le fait qu'ils ne puissent plus exporter leurs biens vers les États de I'UE suite à la décision de la Cour de Justice européenne du mars 1994, qui demande une autorisation du gouvernement de la République de Chypre pour toutes les exportations de Chypre, a porté un grave coup à leur économie ${ }^{64}$. Alors que quelques-uns échappent à cet obstacle en obtenant les autorisations nécessaires de la Turquie ${ }^{65}$, d'autres avancent que même la Turquie n'accepte plus l'exportation de certains biens à cause de cette décision. «Comment l'UE peut-elle expliquer qu'elle exerce un embargo sur les produits de leur pays si elle considère que la partie chypriote turque est aussi une candidate?», reprochent-ils à l'UE ${ }^{66}$.

Cela étant, on attendait que la « carotte » de l'adhésion à l'Union attire les Chypriotes turcs qui sont bien conscients des avantages économiques de l'adhésion. Mais ce n'est pas le cas : le gouvernement de la RTCN nie le besoin des fonds européens. S'ils ont pu survivre jusqu'à maintenant, grâce à la Turquie, ils peuvent continuer à survivre ainsi. «Nous n'avons pas le luxe de multiplier par zéro notre cause pour cet argent», affirme Tahsin Ertuğruloğlu. D'autres sont frustrés de ne pouvoir recevoir cet argent auquel ils ont droit et dont ils ont

\footnotetext{
${ }^{61} 43,67 \%$ des votes se sont portés sur Rauf Denktaş et $30.14 \%$ sur Derviş Eroğlu au premier tour des élections le 15 avril 2000, ce qui nécessitait un deuxième tour d'élection. Alors que les deux candidats rencontraient les dirigeants des partis politiques à la recherche de soutiens, Eroğlu annonçait subitement le 19 avril qu'il se désistait. Le même jour, la Turquie a annoncé qu'elle accorderait un crédit de 100 million $\$$ à la RTCN. Cette manipulation n'a guère surpris les Chypriotes turcs qui y sont habitués, mais le public turc, ayant appris que le discours ana-yavru ne disait pas tout, à travers la presse turque, s'est soudain intéressé à la RTCN.

${ }^{62}$ Une des raisons de la faiblesse de l'économie chypriote turque est l'instabilité de l'économie de la Turquie. Ainsi toutes les crises économiques en Turquie et les dévaluations de la livre turque affectent également la RTCN.

${ }^{63}$ Suite à la faillite des six banques, les titulaires des comptes touchés ont envahi le Parlement chypriote turc, vandalisant les voitures des parlementaires. Ils ont demandé la démission du gouvernement et du Président. Voir le Kıbrıs du 25 juillet 2000 qui est publié avec une page de couverture noire. Selon Varol Öztuğ, le Secrétaire général du KTÖS, tout cela serait un complot organisé par Ankara pour pouvoir justifier un changement de sa politique chypriote qu'elle devrait effectuer pour adhérer à l'UE. Voir Kıbrıs du 10 août 2000.

${ }^{64}$ Les pays de l'UE constituaient son premier marché d'exportation.

65 Le quotidien Kıbrıs annonçait récemment que les agrumes pourraient être d'ores et déjà exportés vers l'UE en obtenant les certificats nécessaires des autorités en Turquie. Voir Kıbrıs du 4 aôut 2000.

${ }^{66}$ Entretien cité avec Hakkı Atun, op. cit.
} 
besoin. II est clair que le gouvernement de la RTCN n'accepte pas ces fonds, qui arrivent à la Banque Centrale de Chypre, dont une partie est destinée à la communauté chypriote turque. Le gouvernement de la République de Chypre n'accepte pas de les transférer directement aux autorités chypriotes turques illégales. Les Chypriotes turcs estiment que I'UE pourrait accepter, par exemple, de transférer cet argent aux autorités onusiennes.

Alors que les dirigeants chypriotes des deux côtés s'obstinent au nom de leurs causes nationales, la perspective d'adhésion à l'UE ne contribue guère aux efforts de réconciliation. La perspective d'adhésion à l'UE a changé les paramètres du problème chypriote. Elle l'a rendu en fait encore plus compliqué, car les représentants de I'Union assistent aux pourparlers. Donc I'UE constitue un acteur de plus dont les intérêts devraient être sauvegardés ${ }^{67}$. De plus, l'UE est perçue par la plupart comme un acteur partial, trop soumis à l'influence des chantages grecs. Tout ce processus d'adhésion et les décisions que l'UE a prises ne motivent pas les Chypriotes grecs car ils sont sûrs que la reconnaissance de leur État va entraîner leur adhésion finalement. L'UE pourrait donc contribuer à la résolution du conflit si elle prenait une position plus ferme en confirmant que l'adhésion de Chypre exige d'abord la résolution du conflit. En effet, si I'UE faisait cela, les Chypriotes turcs lui feraient d'ores et déjà plus confiance.

\section{CONCLUSION}

L'accent mis sur les avantages économiques que les Chypriotes turcs tireraient de l'adhésion à I'UE n'ont pas été suffisants pour les attirer. Certes, ils veulent tout ce que l'Union représente : paix, prospérité, sécurité, droits de l'homme, dépassement des souverainetés nationales. Mais ils ne se sentent guère rassurés par ce discours, car l'histoire du problème chypriote est toujours vivante dans les esprits de beaucoup d'entre eux. La sécurité que les soldats turcs ont apportée à la RTCN est concrète alors que ces valeurs ne semblent qu'abstraites et les Européens paraissent plutôt hypocrites, vu leur comportement en Bosnie et au Kosovo.

Si les Chypriotes grecs semblent avoir saisi l'enjeu de devenir membre de l'Union, c'est grâce à la connaissance de l'UE et de ses institutions depuis le commencement des négociations d'adhésion. L'important est donc d'informer les Chypriotes turcs dont la majorité manque de connaissance en la matière mais qui en demandent toujours plus depuis qu'ils

\footnotetext{
${ }^{67}$ Entretien cité avec Ergün Olgun.
} 
sont les seuls à être en dehors du processus d'adhésion à l'UE, suite à l'acceptation de la candidature de la Turquie au sommet de Helsinki.

Chypre demeure toujours à la tête des pays candidats, ayant provisoirement clôturé 22 des 31 chapitres de négociations. On estime que tous les chapitres seront clos d'ici à $2002^{68}$. Étant donné que le processus d'adhésion de Chypre ne peut être en principe inversé, il appartient à toutes les parties concernées de contribuer à la réunification de l'île, car le nonrèglement de la question chypriote empêche tout progrès à Chypre. Elle pèse aussi bien évidemment sur la question de l'adhésion de Chypre à l'Union européenne. La grande question reste donc toujours de résoudre le problème chypriote.

Or, les derniers efforts visant la résolution du conflit n'ont pas abouti. La démarche a été lancée cette fois-ci par le G8, lors du Sommet de Cologne de juin 1999 où les Etats du G8 ont invité les dirigeants des deux parties à entreprendre des négociations approfondies sans conditions préalables ${ }^{69}$. Cette initiative a été renforcée par la résolution du Conseil de sécurité 1250 (1999), adoptée le 29 juin 1999 où les principes à respecter lors des négociations qui débuteraient en automne 1999 étaient définis ainsi: pas de conditions préalables ; toutes les questions doivent être mises sur la table; engagement de bonne foi de poursuivre les négociations jusqu'à ce qu'un règlement soit trouvé; prise en compte intégrale des résolutions des Nations Unies et des traités de référence. Force est de constater que les négociations indirectes ont une fois de plus échoué.

Lorsque la résolution à Chypre parait de nouveau loin, le scénario de l'adhésion seule des Chypriotes grecs, qui ne semblait qu'un scénario «impossible» ou comme un « cauchemar », devient de plus en plus plausible. Par conséquent, on entend de plus en plus parler, en Turquie, de l'option d'intégrer la RTCN à la Turquie. L'avenir de Chypre reste donc toujours incertain...

\footnotetext{
${ }^{68}$ EU-Cyprus Joint Parliamentary Committee, Press Statement, Nicosie, 18 avril 2000, p. 1.

69 Conclusions de la Réunion des Ministres des Affaires étrangères du G8 à Cologne, 'Guerzenich', le 10 juin 1999, Sommet du G8 de Cologne, 18-20 juin 1999.
} 


\section{SOURCES}

\section{Presse}

(Quotidiens chypriotes turcs)

Avrupa

Kıbrıs

Yeni Demokrat

Yenidüzen

\section{Documents officiels}

(Par ordre chronologique)

\section{Commission européenne}

Avis de la Commission sur la demande d'adhésion de la République de Chypre à la Communauté, 30 juin 1993.

\section{Conseil européen}

Conseil européen de Luxembourg, Conclusions de la Présidence, 12 et 13 décembre 1997. Conseil européen d'Helsinki, Conclusions de la Présidence, 10 et 11 décembre 1999.

Conseil européen de Santa Maria de Feira, Conclusions de la Présidence, 19 et 20 juin 2000.

\section{Parlement européen}

Committee on Foreign Affairs, Human Rights, Common Security and Defence Policy, Rapporteur: Elmar Brok, Report on the Proposal for a Council regulation on the implementation of operations in the Framework of the pre-accession strategy for Cyprus and Malta, 3 février 2000.

\section{Divers}

Résolution 649 (1990) du Conseil de Sécurité du 12 mars 1990.

CHYPRE: La marche vers l'adhésion à I'U.E., Bureau de Presse et d'Information, République de Chypre, 1997. 
Conclusions de la Réunion des Ministres des Affaires étrangères du G8 à Cologne, 'Guerzenich', le 10 juin 1999, Sommet du G8 de Cologne, 18-20 juin 1999.

\section{Sites Internet}

Åland Islands Homepage

<http://www.aland.fi/virtual/fransk/France.html>

Entretiens : (Par ordre chronologique)

- İsmail Bozkurt, Président du Centre des Études chypriotes de la DAÜ (Doğu Akdeniz Üniversitesi, «l'Université de la Méditerranée orientale»), Gazimağusa, 5 avril 2000.

- Ergün Olgun, Conseiller politique du Président de la RTCN, Lefkoşa, 5 avril 2000.

- Ferdi Sabit Soyer, Secrétaire général du CTP, Lefkoşa, 6 avril 2000.

- Önder Konuloğlu, Président de TÜRK-SEN, Lefkoşa, 6 avril 2000.

- Alpay Durduran, Dirigeant du YBH, Lefkoşa, 6 avril 2000.

- Mustafa Damdelen, Membre de la KKTC IŞAD, Lefkoşa, 6 avril 2000.

- Ali Erel, Membre de la KKTC IŞAD, Lefkoşa, 6 avril 2000.

- Fatma Azgın, Pharmacienne, Participante aux activités bicommunautaires, Lefkoşa, 6 avril 2000.

- Sevgül Uludağ, Journaliste au Yenidüzen, Participante aux activités bicommunautaires, Lefkoşa, 7 avril 2000.

- Hakkı Atun, Président honoraire du DP, ancien Premier Ministre et Président du Parlement de la RTCN, Lefkoşa, 7 avril 2000.

- Hasan Ali Bıçak, Professeur, Responsable du Centre d'information et de recherche sur I'Union européenne à la DAÜ, Gazimağusa, 7 avril 2000. 
- Tahsin Ertuğruloğlu, Ministre des affaires étrangères et de la défense de la RTCN, Lefkoşa, 7 avril 2000.

- Ahmet Barçın, Président de KTOEÖS, Lefkoşa, 8 avril 2000.

- Emine Erk, Avocat, Participante aux activités bicommunautaires, Lefkoşa, 8 avril 2000.

- Varol Öztuğ, Secrétaire général du KTÖS, Lefkoşa, 8 avril 2000.

\section{BIBLIOGRAPHIE}

Akıncı (Mustafa), Conférence intitulée «Kıbrıs'ta Çözüm ve Avrupa Birliği», AKM, Lefkoşa, 5 avril 2000.

Bertrand (Gilles), «Vingt-cinq ans après, où en est la partition de Chypre», Les Études du CERI, (59), novembre 1999.

Denktaş (Rauf R.), Kıbrıs Meselesinde Son Durum, Kıbrıs, Raif Denktaş Eğitim Vakfı Yayınlari, 1996.

Diez (Thomas), «Last Exit to Paradise? The EU, the Cyprus Conflict, and the Problematic 'Catalytic Effect'», COPRI (Copenhagen Peace Research Institute) Working Papers, 4/2000.

Dodd (Clement H.), «Political and Administrative Structures», in Clement H. Dodd (ed.), The Political Social and Economic Development of Northern Cyprus, Huntingdon, The Eothen Press, 1993, pp. 167-192. 
Heinze (Dr. Christian), On the question of the compatibility of the admission of Cyprus into the European Union with international law, the law of the EU and Cyprus Treaties of 1959/60, Appraisal study presented to the Republic of Turkey, Munich, mars 1997.

Ioannides (Christos P.), In Turkey's Image: The Transformation of Occupied Cyprus into a Turkish Province, New Rochelle, NY, Caratzas, 1991.

İncirli (Çiler), Bir Daha Asla, Lefkoşa, Kuzey Kıbrıs Türk Cumhuriyeti Dışişleri ve Savunma Bakanlığı, 1998.

Kabaalioğlu (Halûk), "Cyprus and the European Union: A Perspective», in Doğramacı (Emel), Haney (William) et König (Günay) (eds), Proceedings of the First International Congress on Cypriot Studies, Gazimağusa, Eastern Mediterranean University Press, 1997, pp. 219-232.

Mendelson (Maurice H.), EU and Cyprus: An Expert View: opinion on the application of the Republic of Cyprus to join the European Union, Lefkoşa, 1997.

Richarte (Marie-Pierre), La Partition de Chypre. Étude géopolitique en Méditerranée orientale, Thèse de Doctorat sous la direction de Paul Chaval, Université Paris IV, 1995.

Stephen (Michael), The Cyprus Question, London, British-Northern Cyprus Parliamentary Group, 1997.

Tonneau (Corine), Le rapprochement Chypre-Union européenne: l'impact du conflit intercommunautaire, Mémoire de fin d'études sous la direction de Christian Franck, Université Catholique de Louvain, 1999. 
ANNEXES

- Élections présidentielles de la RTCN

\begin{tabular}{|c|c|c|c|c|c|c|c|}
\hline & $\begin{array}{c}20 \text { juin } \\
1976\end{array}$ & $\begin{array}{c}28 \text { juin } \\
1981\end{array}$ & $\begin{array}{l}9 \text { juin } \\
1985\end{array}$ & $\begin{array}{c}22 \text { avril } \\
1990\end{array}$ & $\begin{array}{c}15 \text { avril } \\
1995 \\
1^{\text {er }} \text { tour }\end{array}$ & $\begin{array}{c}22 \text { avril } \\
1995 \\
2^{\mathrm{e}} \text { tour }\end{array}$ & $\begin{array}{c}15 \text { avril } \\
2000\end{array}$ \\
\hline $\begin{array}{l}\text { Rauf R. } \\
\text { Denktaş }\end{array}$ & $76,6 \%$ & $51,7 \%$ & $70,2 \%$ & $66,7 \%$ & $40,4 \%$ & $62,5 \%$ & $43,67 \%$ \\
\hline $\begin{array}{c}\text { A. M. } \\
\text { Berberoğlu } \\
\text { (CTP) }\end{array}$ & $21,8 \%$ & & & & & & \\
\hline $\begin{array}{c}\text { Özker Özgür } \\
\text { (CTP) }\end{array}$ & & $12,7 \%$ & $18,3 \%$ & & $19 \%$ & & \\
\hline $\begin{array}{l}\text { Mehmet Ali } \\
\text { Talat (CTP) }\end{array}$ & & & & & & & $10,03 \%$ \\
\hline $\begin{array}{c}\text { Ziya Rızkı } \\
\text { (TKP) }\end{array}$ & & $30,5 \%$ & & & & & \\
\hline $\begin{array}{c}\text { Alpay } \\
\text { Durduran } \\
\text { (TKP) } \\
\text { (YKP) }\end{array}$ & & & $9,5 \%$ & $1,2 \%$ & $1,8 \%$ & & \\
\hline $\begin{array}{c}\text { Mustafa } \\
\text { Akıncı (TKP) }\end{array}$ & & & & & $14,12 \%$ & & $11,7 \%$ \\
\hline $\begin{array}{l}\text { Derviş } \\
\text { Eroğlu } \\
\text { (UBP) }\end{array}$ & & & & & $24,1 \%$ & $37,5 \%$ & $30,14 \%$ \\
\hline $\begin{array}{c}\text { Turgut } \\
\text { Afşaroğlu }\end{array}$ & & & & & & & $0,56 \%$ \\
\hline $\begin{array}{c}\text { İsmail } \\
\text { Bozkurt }\end{array}$ & & & & $32,1 \%$ & & & \\
\hline $\begin{array}{l}\text { Servet S. } \\
\text { Dedeçay }\end{array}$ & $0,8 \%$ & & $0,7 \%$ & & & & \\
\hline $\begin{array}{l}\text { A. H. T. } \\
\text { Desem }\end{array}$ & & & $0,9 \%$ & & & & $2,6 \%$ \\
\hline $\begin{array}{c}\text { Ayhan } \\
\text { Kaymak }\end{array}$ & & & $0,4 \%$ & & $0,4 \%$ & & $0,38 \%$ \\
\hline $\begin{array}{c}\text { Şener } \\
\text { Levent }\end{array}$ & & & & & & & $0,92 \%$ \\
\hline $\begin{array}{c}\text { autres } \\
\text { (en cumulé) }\end{array}$ & $0,8 \%$ & $4,8 \%$ & - & - & $0,1 \%$ & & - \\
\hline
\end{tabular}

Source: Kıbrıs, 16 avril 2000. 
- Élections à la Chambre des représentants de la RTCN

\begin{tabular}{|l|c|c|c|c|c|c|c|c|c|c|c|c|}
\hline & \multicolumn{2}{|c|}{\begin{tabular}{c}
20 juin \\
\multicolumn{2}{|c|}{1976}
\end{tabular}} & \multicolumn{2}{c|}{$\begin{array}{c}23 \text { juin } \\
1981\end{array}$} & \multicolumn{2}{c|}{1985} & \multicolumn{2}{c|}{6 mai } & \multicolumn{2}{c|}{12 décembre } & \multicolumn{2}{c|}{6 décembre } \\
& $\%$ & sièges & $\%$ & sièges & $\%$ & sièges & $\%$ & sièges & $\%$ & sièges & $\%$ & sièges \\
\hline & 53,7 & 30 & 42,5 & 18 & 36,7 & 24 & 54,7 & 34 & 29,85 & 17 & 40,3 & 24 \\
\hline UBP & 20,2 & 6 & 15,1 & 6 & 21,4 & 12 & & & 24,16 & 13 & 13,4 & 6 \\
\hline CTP & 12,9 & 2 & 28,5 & 13 & 15,8 & 10 & 44,5 & 16 & 13,27 & 5 & 15,4 & 7 \\
\hline TKP & & & & & & & 0,8 & - & 1,2 & - & 2,6 & - \\
\hline YKP & 11,7 & 2 & 8,1 & 2 & 7,4 & - & & & & & & \\
\hline DHP & & & 5,5 & 1 & & & & & & & & \\
\hline TBP & & & & & 8,8 & 4 & & & & & & \\
\hline YDP & & & & & & & & & 29,19 & 15 & 22,6 & 13 \\
\hline DP & & & & & & & & & & & \\
\hline
\end{tabular}

UBP (Ulusal Birlik Partisi) ; CTP (Cumhuriyetçi Türk Partisi) ; TKP (Toplumcu Kurtuluş Partisi) ; YKP (Yeni Kıbrıs

Partisi) ; DHP (Demokrat Halk Parti) ; TBP (Türk Birliği Partisi) ; YDP (Yeni Doğuş Partisi) ; DP (Demokrat Parti)

Note:

1) Le HP (Halkçı Parti) devient DHP en 1979.

2) Le CTP et le TKP ont formé une coalition électorale en 1990.

3) Le YKP (Yurtsever Birlik Hareketi) est devenu YBH en 1998.

Source: Bertrand (Gilles), "Vingt-cinq ans après, où en est la partition de Chypre ", Les Études du CERI, (59), novembre 1999.

Article tiré des

Cahiers d'études sur la Méditerranée orientale et le monde turco-iranien,

$N^{\circ} 31$, janvier-juin 2001 